\title{
The Detection of Normal and Epileptic EEG Signals using ANN Methods with Matlab-based GUI
}

\author{
Gamze Doğalı Çetin \\ Sakarya University \\ Department of Computer and \\ Information Sciences
}

\author{
Özdemir Çetin \\ Sakarya University \\ Department of Electrical and \\ Electronic Engineering
}

\author{
Mehmet Recep Bozkurt \\ Sakarya University \\ Department of Electrical and \\ Electronic Engineering
}

\begin{abstract}
Epilepsy is common neurological disorder disease in the world. Electroencephalogram (EEG) can provide significant information about epileptic activity in human brain. Since detection of the epileptic activity requires analyzing of very length EEG recordings by an expert, researchers tend to improve automated diagnostic systems for epilepsy in recent years. In this work, we try to automate detection of epilepsy using EEG based on Matlab Graphical User Interface (GUI). Three different types of Artificial Neural Networks (ANN), namely, Feed Forward Backpropagation, Cascade and Elman neural networks, are used for the classification EEG (existence of epileptic seizure or not). Before classification process, we use autoregressive model to data reduction and three different AR model algorithms to calculate the coefficients. Developed Matlab-based GUI provides flexible and visual utilization to observe normal/epileptic EEG and test results. Training parameters and type of neural networks are decided by users on the interface. Performance of the proposed model is evaluated using overall accuracy.
\end{abstract}

\section{General Terms}

Artificial Neural Networks, Matlab Graphical User Interface, and Biomedical Signal Processing

\section{Keywords}

EEG Signals, Artificial Neural Network, Epilepsy, Matlab Graphical User Interface

\section{INTRODUCTION}

Epilepsy is a neurological disease which appears in approximately one percentage of the world's population [1]. Brain activity normally leads to low amplitude electrical signals in the human brain. Epilepsy results from excessive and uncontrolled permeation of these electrical signals in the brain. The main characteristic of epilepsy is irregular and unpredictable seizure. The seizure can cause loss of consciousness, physical injuries, and also death. Thus, detection and diagnosis of epilepsy become more of an issue. Electroencephalography (EEG) is recording of electrical potential difference during brain activity. EEG signals are commonly used for observation of brain activity and diagnosis of neurological disorders [2]. EEG signals contain very important information for understanding epilepsy [3]. In the literature there are various approaches for automatic detection of epilepsy using EEG signals. L. Guo et al [3] proposed automatic epileptic seizure detection using line length features based on wavelet transform. Kumar et al [4] have demonstrated detection of epilepsy focusing on entropy, Elman and Radial based neural network models. Şahin et al [5] performed the partial epilepsy detection using Multiplayer Perceptron Neural Networks (MLPNNs). Orhan et al [6] classified epileptic seizure segments using Wavelet
Transform, K-means clustering algorithm, and Multiplayer Perceptron Neural Networks (MLPNN).

In this work, we designed a system to detect epilepsy based on artificial neural network. Used EEG dataset contain epileptic and healthy EEG signals. The EEG signals waveform can be observed over the user friendly Matlab GUI. In preprocessing step, Auto Regression methods reduce dataset to decrease processing time. We used three different parametric methods, such as Burg Algorithm, Yule Walker Method, and Covariance Method, so users can compare their performances. Next step, the classification of EEGs is done using ANNs such as Feed-Forward Backpropagation Network, CascadeForward Backpropagation Network, and Elman Backpropagation Network. The designed Matlab GUI provides to put in ANN's parameters, Epoch Number, Goal Number, and Learning Rate, to users. User friendly Matlab GUI also offers easy and flexible usage to get the best classification results detecting epilepsy.

The paper is structured as follows. In Section 2, EEG signals, ANN and Autoregression methods are briefly described. In Section 3, experimental work and performance evaluation are presented. Finally, the results of the proposed study is discussed.

\section{MATERIALS AND METHODS}

\subsection{Electroencephalography (EEG)}

Electroencephalography or EEG is a method which provides monitoring of brain neural activity using electrical signals. [7]. EEG provides information about functional state of brain more than structural functions. EEG signals are recorded by electrodes placed over the head. Placements of electrodes are shown in Figure 1 [8].
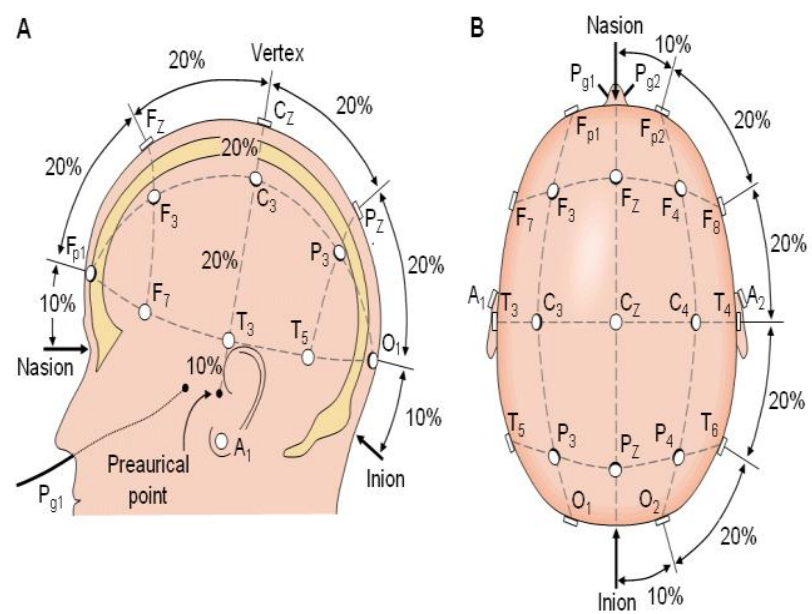

Fig 1: Electrode settlement for measuring EEG signals

Frequency spectrum of EEG signals range is between $0.5-$ 
$100 \mathrm{~Hz}$. EEG signals are divided into four groups according to frequency spectrum [9]. EEG signals with their four rhythms are shown in Figure 2 [10]. Figure 2 also shows that brain mental activity in these frequency values.

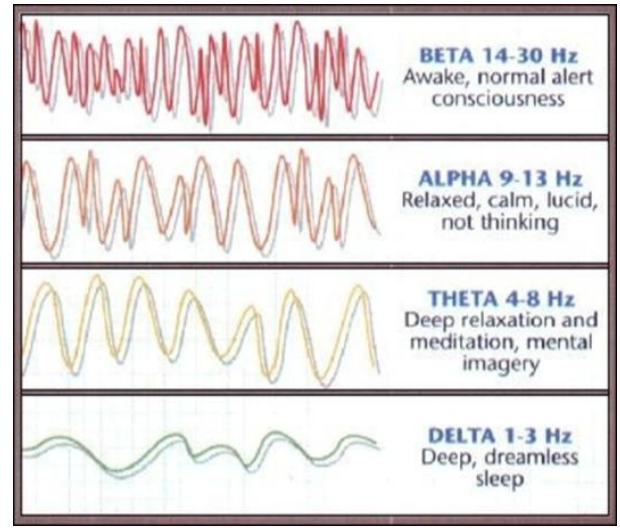

Fig 2. EEG signals in frequency spectrum

The amplitude of EEG signals range is between 1-100 $\mu \mathrm{V}$ [11]. But the amplitude of EEGs increases excessively in seizure activity in epilepsy subjects. Thus, capturing extended amplitude changes is a reasonable approach to detect epilepsy.

EEG signals amplitude and frequency are shown in Figure 3 [12] for epilepsy subjects. The amplitude and frequency values increase dramatically in pre-seizure and during the seizure activity as is seen in Figure 3.

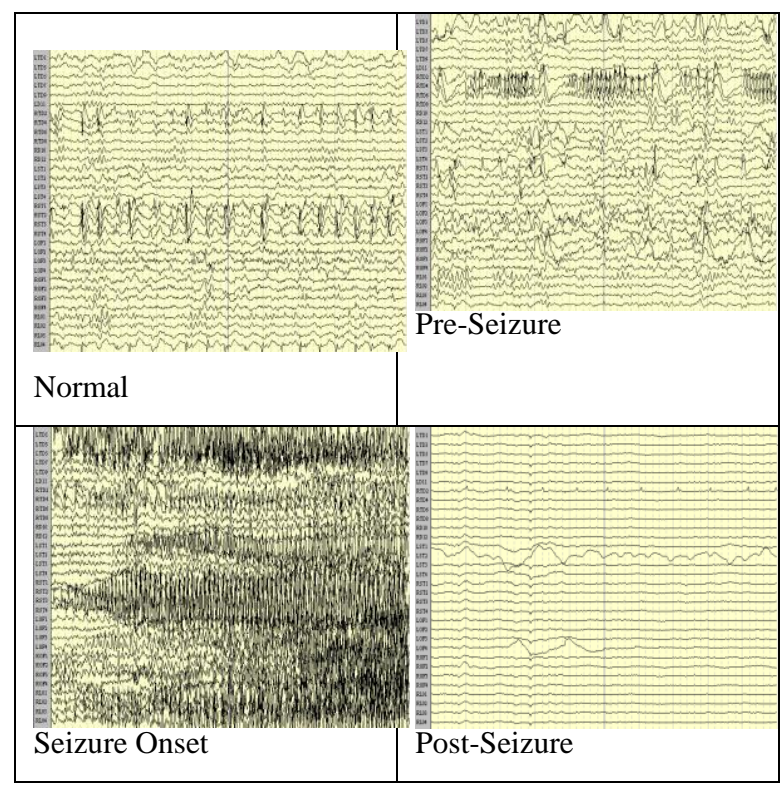

Fig 3: Frequency and amplitude values for epileptic EEG signals

\subsection{Artificial Neural Network Classifier}

Artificial Neural Network (ANN) is designed to achieve process like a human brain. ANN is a mathematical model which perform calculating, making decision and learning. This method based on human biological nervous system, thus ANN components simulate biological neural network. In an ANN, processing element, weight, add function, activation function and exit correspond respectively to neuron, synapse, dendrite, cell body, and axon in a biological neural network [13]. In Figure 4, a matching of biological and artificial neural network is given [14].

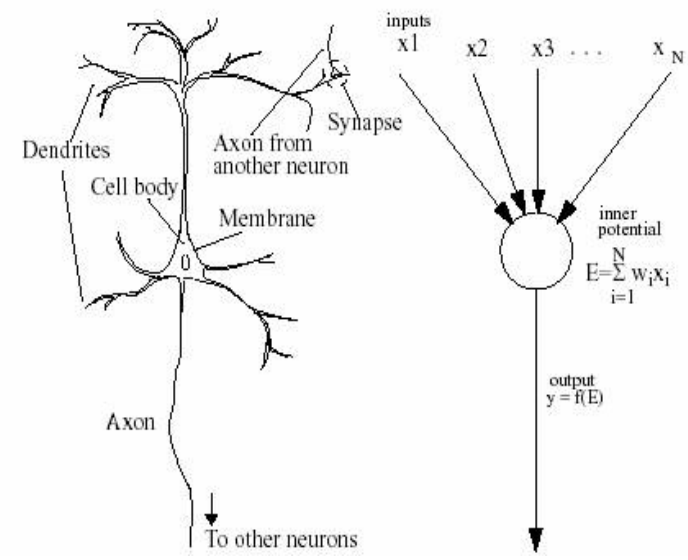

Fig 4: A Matching of Biological and Artificial Neural Network

ANN is grouped as Supervised Learning, Unsupervised Learning, and Reinforcement Learning according to learning paradigm. ANN is also categorized into two groups with regard to neurons architectural structure, namely FeedForward Neural Networks and Feed-Back Neural Networks.

Another classification for ANN is based on learning time. Statistical Learning and Dynamic Learning are used for these types of ANN [15].

In this work, we used three different ANN types, namely Feed-Forward Backpropagation Neural Network, Elman Neural Network, and Cascade Neural Network. The users can choose of them from designed user interface and compare each other regarding their speed, error rate, parameters requirement, and ease of use. In addition, the Matlab-based GUI visually presents the training results and error rate.

\subsection{Autoregressive (AR) Method}

Autoregressive method is an alternative approach for modeling the spectrum of signals [16]. In this method, AR coefficients can calculate different methods. In this work, three different methods; Yule Walker, Burg Algorithm, and Covariance Method is used to calculate $a_{p}(k)$ coefficients. For Yule Walker method, autocorrelation equations are as following,

$$
\left[\begin{array}{ccccc}
r_{x}(0) & r_{x}(1) & r_{x}(2) & \cdots & r_{x}(p) \\
r_{x}(1) & r_{x}(0) & r_{x}(1) & \ddots & r_{x}(p-1) \\
& \vdots & & \cdots & \vdots \\
r_{x}(p) & r_{x}(p-1) & r_{x}(p-2) & r_{x}(0)
\end{array}\right]\left[\begin{array}{c}
1 \\
a_{1} \\
\vdots \\
a_{p}
\end{array}\right]=\varepsilon_{p}\left[\begin{array}{c}
r_{x}(0,1) \\
r_{x}(0,2) \\
\vdots \\
r_{x}(0, p)
\end{array}\right]
$$

Here, $r_{x}(k)$ is calculated by following equation,

$$
r_{x}(k)=\frac{1}{N} \sum_{n=0}^{N-1-k} x_{n+k} x_{k}^{*} ; \quad k=0,1, \ldots, p
$$

then,

$$
\left|b_{0}\right|^{2}=\varepsilon_{p}=r_{x}(0)+\sum_{k=1}^{p} a_{k} r_{x}^{*}(k)
$$

is obtained.

Second method Burg Algorithm is similar with Yule Walker method. While Yule Walker method $x_{n}$ components are accepted as autoregression process, Burg Algorithm $x_{n}$ components are accepted as Gaussian. 
Covariance Method as third method, autocorrelation equations $r_{x}(k)$ matrix are differently calculated from Yule Walker method.

$\left[\begin{array}{ccccc}r_{x}(1,1) & r_{x}(2,1) & r_{x}(3,1) & \cdots & r_{x}(p, 1) \\ r_{x}(1,2) & r_{x}(2,2) & r_{x}(3,2) & \ddots & r_{x}(p, 2) \\ & \vdots & & \cdots & \vdots \\ r_{x}(1, p) & r_{x}(2,1) & r_{x}(3,1) & & r_{x}(p, p)\end{array}\right]\left[\begin{array}{c}a_{1} \\ a_{2} \\ \vdots \\ a_{p}\end{array}\right]=-\left[\begin{array}{c}r_{x}(0,1) \\ r_{x}(0,2) \\ \vdots \\ r_{x}(0, p)\end{array}\right]$

Where,

$$
r_{x}(k, 1)=\sum_{n=p}^{N-1} x_{n-1} x_{n-k}^{*}
$$

is obtained.

In this work, these methods used for parameter estimation corresponding to AR parametric signal model, thus big EEG data effectively reduced in order to perform ANN input.

\section{EXPERIMENTAL WORK}

\subsection{Proposed System}

Block diagram of the proposed system is given in Figure 5. First step in proposed work is data acquisition. Used two sets of EEG data, normal and epileptic EEG signals, were gained from 100 healthy and 100 epilepsy subjects. One subject record has 4096 samples of one EEG time series. EEG data sets obtained from epilepsy center of University of Bonn, Germany $[17,18]$.

In the second step, data reduction was performed with $\mathrm{AR}$ methods. One of the Yule-Walker method, Burg Algorithm, and Covariance AR methods can be selected on the designed Matlab GUI. EEG records of each subject reduced from 4096 to 8 by these methods, before training.

In third step, ANN was used for training and classification. Among the EEG data sets, totally 140 subjects' EEG records, 70 healthy and 70 epileptic, were used for ANN training. Then the remaining 60 subjects' EEG records, 30 healthy and 30 epileptic were given to the trained network as test data. All process, plotting EGG signals, training and classification, simulation, and the test results are carried out Matlab GUI. The designed system is evaluated using overall accuracy performance parameter.

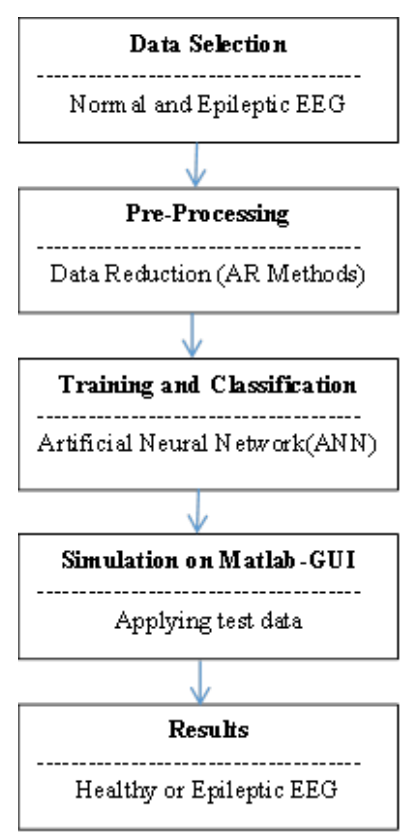

Fig 5: Block Diagram of the Proposed System

\subsection{Data Selection}

In this paper, the EEG dataset is selected from Andrzejak et. al's study which is about analysis of brain electrical activity [18]. They collected five sets of EEG data which are indicated A-E. The each of data sets includes 100 single-channel EEG signals recorded during $23.6 \mathrm{sec}$. [17, 18]. In set A, healthy volunteers were in awake and relaxed position with open eyes, while in set $\mathrm{B}$, in the same case volunteers were with closed eyes. Sets C, D, and E obtained from presurgical diagnosis archive. Sets C and D include EEG data which were collected during seizure free intervals, but data set E contains EEG signals during seizure activity [17, 18]. Signal acquisition systems' EEG time series spectral bandwidth, sampling rate, and band-pass filter settings are $0.5 \mathrm{~Hz}$ to $85 \mathrm{~Hz}$., $173.61 \mathrm{~Hz}$., and $0.53-40 \mathrm{~Hz} .(12 \mathrm{~dB} / \mathrm{oct})$, respectively $[17,18]$.

In this study, set A and E were used to classify healthy and epileptic EEG signals. Figure 6 shows healthy and epileptic EEG signals on the part of designed Matlab GUI. Users enter subject number to Subject Number edit box, and then EEG signals are plotted on the window pushing Show EEG button. Related signals are loaded with 'Load' button from the recorded file before plotting process.

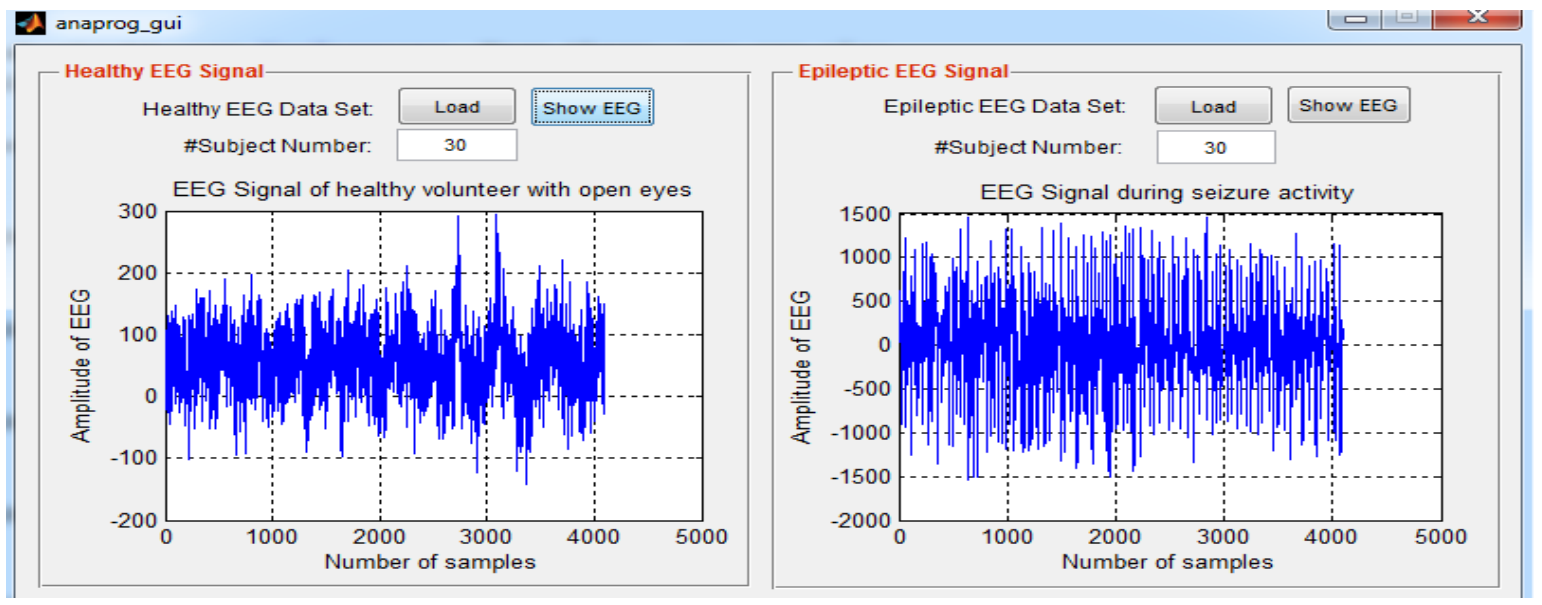

Fig 6: (Left) EEG signals of healthy volunteer with open eyes. (Right) EEG signals during seizure activity 


\subsection{Results}

In designed Matlab GUI consists of two parts. In first part, healthy and epileptic EEG signals are plotted with 'Show EEG' button according to the selected subject number. Before this process, recorded EEG datasets are loaded to Matlab platform with 'Load' button. As is seen in Fig 6, healthy subject's EEG signals' amplitude range varies between $200 \mathrm{mV}$ and $300 \mathrm{mV}$. However, for epileptic subject during seizure activity the same values are observed between $1500 \mathrm{mV}$ and $1500 \mathrm{mV}$.

The second part includes training, classification, and test results. Before the network training, EEG dataset which has 4096 samples for each subject reduced to 8 reasonable data with AR methods. Users can select three types of AR methods, such as Yule-Walker method, Burg Algorithm, and Covariance method from 'Regression Type' popupmenu.

After regression process, the neural network type select from 'ANN Type' popupmenu and the network parameters are input to the related edit boxes. The 'ANN Type' popupmenu includes three types of neural networks; Feed-Forward Backpropagation, Elman Backpropagation, and Cascade Backpropagation.

As shown in Figure 7, Burg Algorithm and Feed- Forward Backpropagation neural network are selected to experimental study. The network parameters; epoch number, goal number, and learning rate are selected as 3000, 0.004, and 0.008 respectively. 'Train' button is used to train the network. Then test data is classified as healthy or epileptic result after pushing the 'Test' button. While level ' 0 ' indicates epileptic case, level ' 1 ' indicates healthy case.

According to the test result among 60 subjects, 30 subjects are determined as healthy and 29 subjects are determined as epilepsy. Thus only one wrong result determined. However 50. subject is epilepsy, according to the test results the subject is healthy. The designed Matlab GUI provides to monitor classification on the graphic window. After all, 'Clear' button makes to remove all parameter values, and clear the graphic.

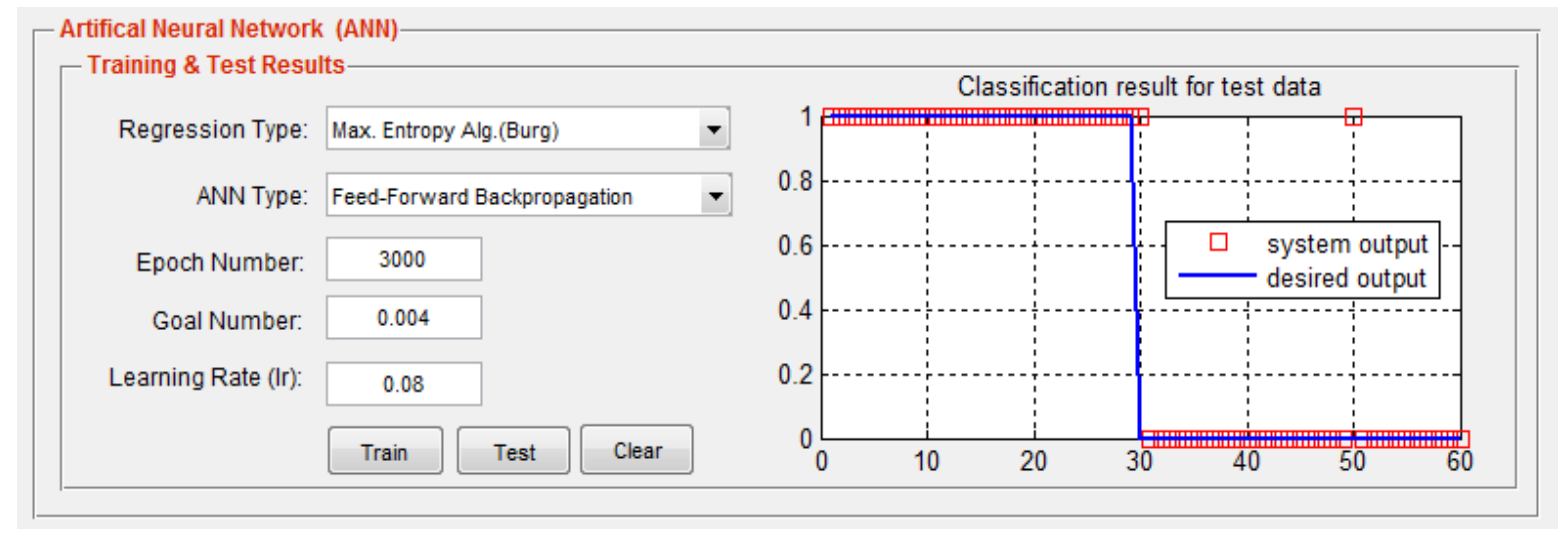

Fig 7: Training and Test Results

To performance evaluation of the three different neural networks, the same scenario performed for each of them. A performance evaluation criterion, Overall Accuracy rate calculated for the used network types. Overall Accuracy (OA) is defined as following:

$$
O A(\%)=\frac{T N_{C D P}}{T N_{A P P}} x 100
$$

Where $T N_{C D P}$ represents the total number of correctly detected samples and $T N_{A P P}$ represents the total number of all patterns.

According to the Table 1, Overall accuracy rate of FeedForward network is the best.

Table1. Overall Accuracy (OA) rates for the ANN classifiers

\begin{tabular}{|l|l|l|l|}
\hline Classifier & $\begin{array}{l}\text { Feed-Forward } \\
\text { Network }\end{array}$ & $\begin{array}{l}\text { Elman } \\
\text { Network }\end{array}$ & $\begin{array}{l}\text { Cascade } \\
\text { Network }\end{array}$ \\
\hline $\begin{array}{l}\text { Overall } \\
\text { Accuracy } \\
\text { (OA) }\end{array}$ & $\% 98.3$ & $\% 96.6$ & $\% 96.6$ \\
\hline
\end{tabular}

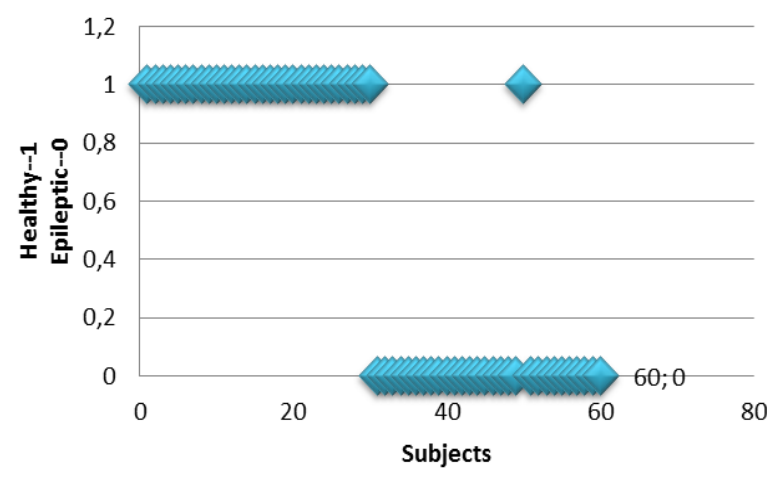

Fig 8: Feed-Forward Network Test Results for 3000 epochs

In Figure 8, Feed-Forward network test result is graphically shown. Level 1 indicates healthy EEGs and level 0 indicates epileptic EEGs. As shown in Figure 8, among 60 subjects 31 subjects are detected as healthy and 29 subjects are detected as epilepsy. However, expected values were 30 healthy and 30 epileptic subjects. Thus, Feed-forward neural network performed epilepsy detection with \%98.3 accuracy rate.

In Figure 9, the designed Matlab GUI is shown. 


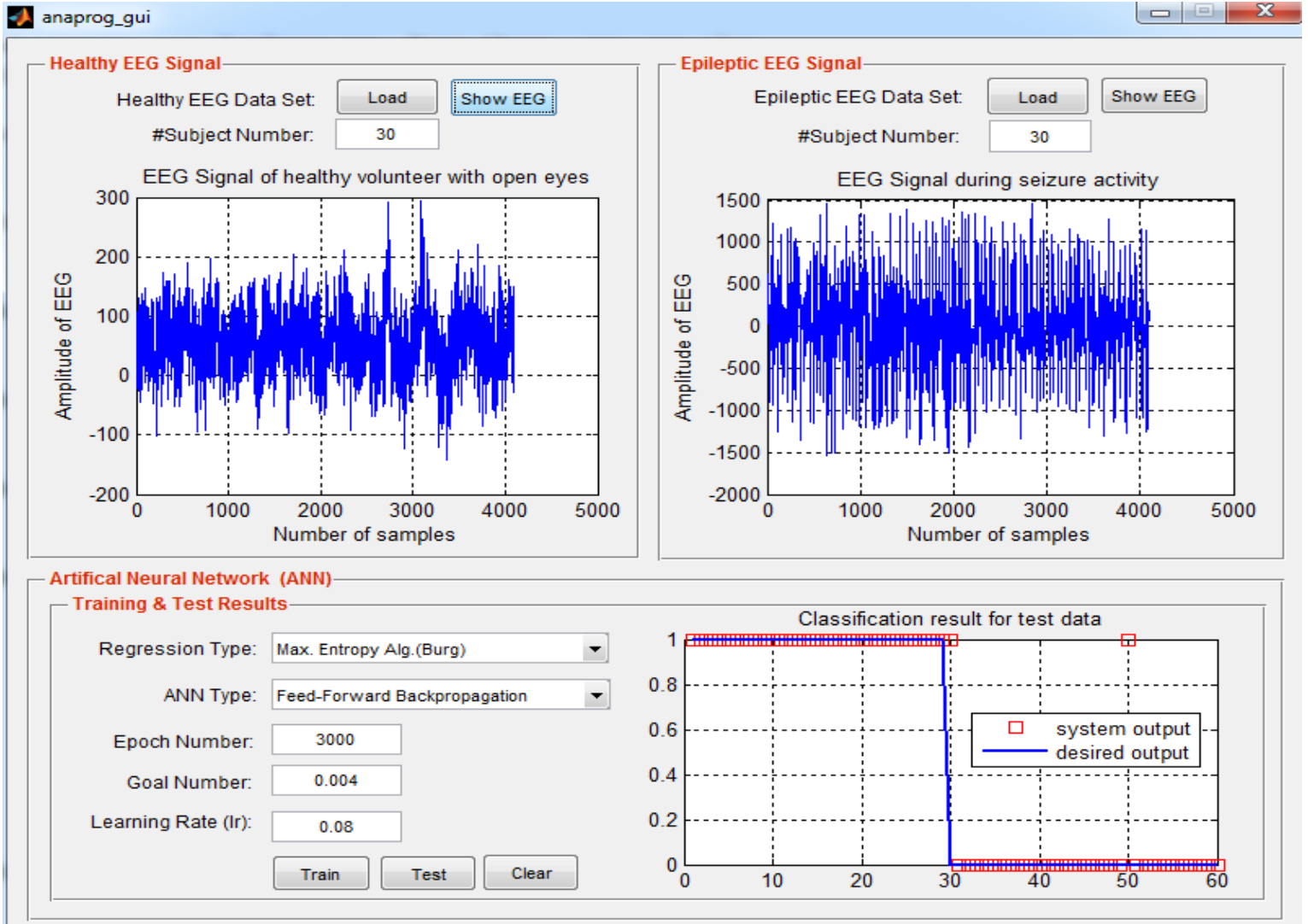

Fig 9: Designed Mablab-based user interface and training/test results

\section{CONCLUSIONS}

Epilepsy is a neurological disease, which appears in approximately one percentage of the world's population. In this study, a new diagnostic system is proposed to follow the medical conditions of the epilepsy people. The aim of the work is to develop an automated diagnostic system for epilepsy diseases based on MATLAB GUI. Increasing confidence of the results, three different types of ANN; Feed

Forward Backpropagation, Cascade and Elman neural networks, were used for EEG classification. And another goal of the study is to develop an ease-of-use MATLAB-based graphical user interface. According to experimental results, the proposed diagnostic approach is accomplished as \%98.3. And also, ease and flexible use of the designed MATLAB GUI provides to analyses of EEG signals even the user is not a medical personnel.

As a future work, the developed MATLAB GUI can be controlled over the Internet by medical person in a health center. Achieving the Internet control, the developed GUI can be designed using a .NET platform for web interface, MATLAB Web Figure for graph analysis, and Microsoft Access for database processing. Thanks to the Web Figure provides a significant benefit such as detailed analysis of signals.

\section{REFERENCES}

[1] Adeli H., Zhou Z., Dadmehr N., (2003), "Analysis of EEG records in an epileptic patient using wavelet transform", Journal of Neuroscience Methods, ,123, 6987.

[2] Agarwal, R., Gotman, J., Flanagan, D., \& Rosenblatt, B. (1998). "Automatic EEG analysis during long-term monitoring in the ICU". Electroencephalography and
Clinical Neurophysiology 107, 44-58.

[3] Guo L.,Rivero D., Dorado J., Rabu nal J.R., Pazos A.,(2010) "Automatic epileptic seizure detection in EEGs based on line length feature and artificial neural networks", Journal of NeuroScience Methods, 191,101109.

[4] Kumar S.P, Sriraam N., Benakop P. G., Jinaga B.C., 2010, "Entropies based detection of epileptic seizures with artificial neural network classifiers", Expert Systems with Applications, 37, 3284-3291

[5] Şahin C., Ogulata S.N., Aslan K., Bozdemir H., Erol R., 2008, "A Neural Network-Based Classification Model for Partial Epilesy by EEG Signals", International Journal of Pattern Recognition and Artificial Intelligence, Vol. 22, No. 5, pp. 973-985.

[6] Orhan U., Hekim M., Ozer M., 2011, "EEG signals classification using the K-means clustering and a multilayer perceptron neural network model”, Expert Systems with Applications, 38, 13475-13481

[7] Batar H., 2007, "Analysis Of EEG Signals Using The Wavelet Transform And Artificial Neural Network", M.Sc. Thesis, Süleyman Demirel University Graduate School of Applied and Natural Sciences, Isparta, Turkey.

[8] http://www.diytdcs.com/tag/1020-positioning, 29.01.2015

[9] Yazgan E., Korürek M., 1995. "Tip Elektroniği”, İstanbul, Turkey.

[10] http://www.earthzense.com/rem-sleep.html, 29.01.2015

[11] Eksi Z., Akgul A.,Bozkurt M.R., 2013,"The 
Classification of EEG Signals Recorded in Drunk and Non-Drunk People",International Journal of Computer Applications (0975-8887), Vol. 68, No. 10.

[12] http://www.slideshare.net/syedirshadmurtaza/ilaeclassification-of-seizures-by-murtaza, 29.01.2015

[13] Vatansever F., Doğalı G., 2011, "Klasik Enterpolasyon Yöntemleri ve Yapay Sinir Ağı Yaklaşımlarının Karşılaştırılması", 6th International Advanced Technologies Symposium (IATS'11), pp. 51-54.

[14] Negishi, M. 1998, "Everything that Linguists have Always Wanted to Know about Connectionism", Department of Cognitive and Neural Systems, Boston University.
[15] Elmas Ç., 2003, Yapay Sinir Ağları, Seçkin Yayıncılık, Ankara.

[16] http://www.itl.nist.gov/div898/handbook/pmc/section4/p mc444.htm, 29.01.2015

[17] EEG time series are available under http://epileptologiebonn.de/cms/front_content.php?idcat=193\&lang=3, 01.12.2014

[18] Ralph K.L., Andrzejak G., Mormann F., Rieke C., David P., Elger C.E., 2001, "Indications of nonlinear deterministic and finite-dimensional structures in time series of brain electrical activity: Dependence on recording region and brain state", Physical Review E, 64, 061907-1-061907-8 